\title{
A Mechanism of Crystallization of Cometary Silicates
}

\author{
Tetsuo Yamamoto \& Takeshi Chigai \\ Department of Earth and Planetary Sciences, Nagoya University, \\ Nagoya 464-8602, Japan
}

\begin{abstract}
We propose a new mechanism of crystallization of cometary silicate. The crystallization is due to heat released by chemical reactions in the organic refractory mantle of Greenberg's model of cometary dust induced by moderate heating by solar radiation. It is shown that the strength of the crystalline feature observed in the comets can be reproduced by the present mechanism of crystallization.
\end{abstract}

\section{Introduction}

Since the first identification of crystalline silicate feature in Comet Halley in the wavelength region of 8 to $13 \mu \mathrm{m}$ (Bregman et al. 1987; Campins \& Ryan 1989), the crystalline silicate features have been observed in dust in several other comets as well as in dust in various kinds of objects (see Hanner 1999 for a review). It has been proposed that cometary crystalline silicate was formed by annealing of amorphous silicate or direct condensation in a hot $(>1000 \mathrm{~K})$ region of the primordial solar nebula. On the other hand, the molecular composition observed in cometary comae requires cold amorphous dust preserving interstellar volatile composition in its icy mantle. To reconcile both requirements, it has been considered that cometary silicates are a mixture of crystalline and amorphous silicates, each of which are of different origin.

In this article, we propose a new mechanism that makes both of the preservation of interstellar volatiles and the presence of crystalline silicate possible without mixing.

\section{The Crystallization Mechanism}

The crystallization mechanism proposed here is based on the Greenberg model of cometary dust (Greenberg 1982; Greenberg \& Zhao 1988), which is composed of a silicate core, an organic mantle and an outer icy mantle. When the dust is released as a comet approaches the Sun, the icy mantle sublimes quickly and the remaining organic mantle and silicate core are heated to temperatures of several hundred $\mathrm{K}$ depending on the heliocentric distance. Note that the composition of the icy mantle preserved the interstellar composition. Although the dust temperatures of several hundred $\mathrm{K}$ are insufficient to crystallize amorphous silicate itself, it will trigger reactions among reactive molecules in the organic mantle because of the increase in the reaction rates due to the temperature rise. The energy released will heat the surface of the silicate core and flows into the 

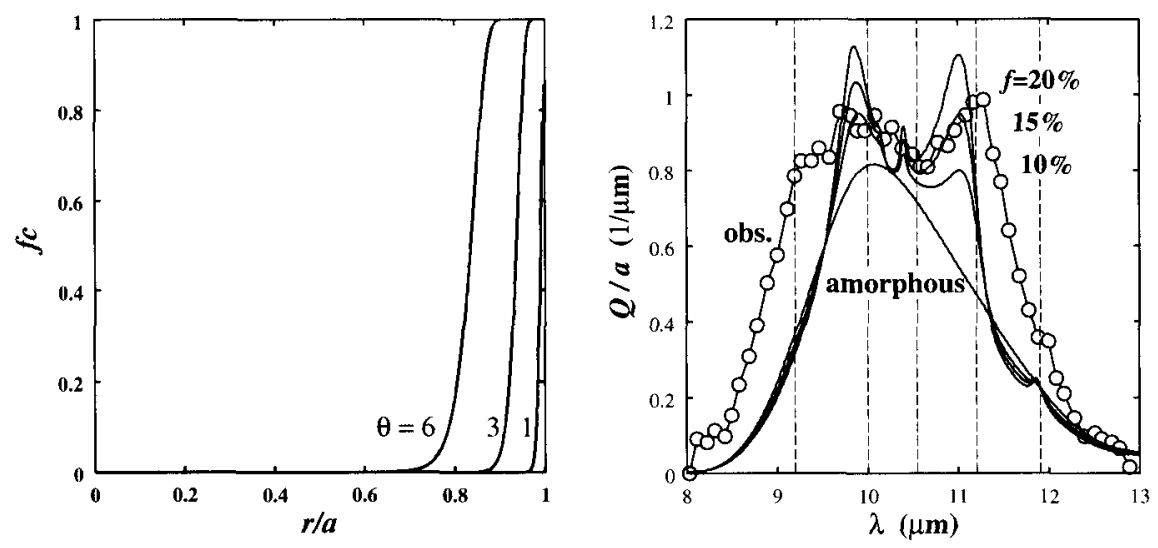

Figure 1. (Left) Degree of crystallinity $f_{c}$ as a function of distance $r$ from the center of a silicate core of radius $a$. See text for $\theta$.

Figure 2. (Right) Comparison of the emissivity spectra of partially crystallized silicate grains with that of Comet Halley (Campins \& Ryan 1989). The volume fraction of crystalline region is denoted by $f$.

core. This leads to crystallization of the amorphous silicate core from the surface towards the interior.

\section{Results}

The crystallization process proposed above is described by the rate equation of crystallization (Haruyama et al. 1993) and by the equation of heat conduction in an originally amorphous silicate core together with the initial condition of the instantaneous heat source on the silicate core surface. In the present model, the degree of crystallinity is determined by only one parameter $\theta$ defined by $\theta=$ $k \Omega n_{\mathrm{r}} h_{\mathrm{r}} E_{\mathrm{r}} / c_{\mathrm{p}}(\pi \chi A)^{1 / 2} E_{\mathrm{c}}$, where $n_{\mathrm{r}}$ is the number density of reactive molecules in the organic refractory mantle of thickness $h_{\mathrm{r}}, E_{\mathrm{r}}$ is the amount of energy released per reaction, $\chi$ is thermal diffusivity of amorphous silicate, $A$ is a time constant on the order of a period of lattice vibration, $c_{\mathrm{p}}$ is specific heat per unit cell, and $E_{\mathrm{c}}$ is activation energy of crystallization of amorphous silicate. The quantity of the largest uncertainty is $n_{\mathrm{r}}$, which is estimated to be $10^{21}$ to $10^{22} \mathrm{~cm}^{-3}$ corresponding to the concentrations of reactive molecules of 1 to $10 \%$ in the organic refractory. Possible values of $\theta$ are then estimated to be 0.6 to 6 . Figure 1 shows the crystallization degree as a function of radial distance from the center of an originally amorphous silicate core, indicating that substantial crystallization occurs near the silicate core surface if $\theta>1$. The volume fraction of the crystallized region in the silicate core is 2 to $40 \%$ for $0.6 \leq \theta \leq 6$.

Does this degree of crystallinity explain the observed strength of the crystalline silicate feature? We focus on the strength of the major peaks at 10.0 and $11.2 \mu \mathrm{m}$ due to crystalline olivine, and make Maxwell-Garnett calculations of the infrared spectra of the partially crystallized silicate grains (Greenberg et al. 1996). We used the complex refractive indices given by Scott \& Du- 
ley (1996) for amorphous forsterite and those by Sogawa et al. (1999) for crystalline forsterite. The spectra for various volume fractions of the crystalline region are compared with the observed spectrum of Comet Halley (Campins \& Ryan 1989) in Fig. 2 in terms of the emissivity $Q$. It is seen that the observed strengths of the main peaks of forsterite at 10.0 and $11.2 \mu \mathrm{m}$ are realized for the crystalline volume fraction around 10 to $20 \%$ in volume. The crystalline fraction that fits best to the observed spectra is about $15 \%$. Note that these crystalline fractions are in the range of those $(2$ to $40 \%)$ expected from the present mechanism of crystallization.

\section{Concluding Remarks}

We have proposed a chemical heating model as a mechanism of crystallization of cometary silicate, and have shown that the chemical heating mechanism leads to the crystallization degree needed to explain the observed strength of the cometary crystalline features. It should be pointed out that the present crystallization mechanism needs neither high temperature to crystallize amorphous silicate nor mixing of amorphous and crystalline silicates, and furthermore can preserve volatiles of the interstellar composition. The mechanism will work for crystallization of grains in the objects other than comets as well as those in protoplanetary disks, if the $\theta$-values of the grains there are large enough to induce crystallization and if moderate heating is available to trigger the chemical heating in the organic refractory mantles on the amorphous silicate cores. The experimental work is hoped to examine the present model.

Acknowledgments. We wish to dedicate this paper to the late Professor J. M. Greenberg, who encouraged us at an early stage of the present study.

\section{References}

Bregman, J. D., Campins, H., Witteborn, F. C., Wooden, D. H., Rank, D. M., Allamandola, L. J., Cohen, M., \& Tielens, A. G. G. M. 1987, A\&A, 187, 616

Campins, H., \& Ryan, E. V. 1989, ApJ, 341, 1059

Greenberg, J. M. 1982, in Comets, ed. L. L. Wilkening, (Tucson: Univ. Arizona Press), 131

Greenberg, J. M., \& Zhao, N. 1988, Nature, 331, 124

Greenberg, J. M., Li, A., Yamamoto, T., \& Kozasa, T. 1996, in ASP Conf. Ser., 104, Physics, Chemistry, \& Dynamics of Interplanetary Dust, eds. B. §. S. Gustafson \& M. S. Hanner, (San Francisco: ASP), 497

Hanner, M. S. 1999, Space Sci.Rev., 90, 99

Haruyama, J., Yamamoto, T., Mizutani, H., \& Greenberg, J. M. 1993, J. Geophys. Res., 98, 15079

Scott, A., \& Duley, W. W. 1996, ApJS, 105, 401

Sogawa, H., Kozasa, T., Koike, C., \& Suto, H. 1999, in Proc. ISAS Lunar Planet. Symp., 32, eds. H. Mizutani \& M. Kato, (Japan; ISAS), 179 